Journal of Advanced Research in Fluid Mechanics and Thermal Sciences

Journal homepage: www.akademiabaru.com/arfmts.html ISSN: 2289-7879

\title{
Effects of Radiation and Magnetohydrodynamic on Unsteady Casson Fluid Over Accelerated Plate
}

\author{
Nur Fatihah Mod Omar ${ }^{1}$, Husna Izzati Osman ${ }^{1}$, Ahmad Qushairi Mohamad ${ }^{2}$, Rahimah Jusoh ${ }^{1}$,

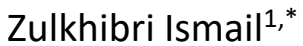 \\ 1 Centre for Mathematical Sciences, College of Computing and Applied Sciences, Universiti Malaysia Pahang, Lebuhraya Tun Razak, 26300 \\ Gambang, Pahang, Malaysia \\ 2 Department of Mathematical Sciences, Faculty of Science, Universiti Teknologi Malaysia, 81310 Johor Bahru, Johor, Malaysia
}

\section{\begin{tabular}{l} 
ARTICLE INFO ABSTRACT \\
\hline
\end{tabular}}

\section{Article history:}

Received 4 May 2021

Received in revised form 3 June 2021

Accepted 10 June 2021

Available online 28 July 2021

\section{Keywords:}

Radiation; magnetohydrodynamics; accelerated plate; Casson fluid

\begin{abstract}
The effects of radiation and magnetohydrodynamic on unsteady Casson fluid through an accelerated plate is analysed. The problem is formulated in the form of Partial Differential Equations (PDE) with imposed initial and boundary conditions. The Partial Differential Equations are transformed into dimensionless form by introducing suitable non-dimensional variables. Laplace transform method is used to derive the exact solutions for temperature and velocity profiles, fulfilling all initial and boundary conditions. The effects of parameters are depicted and illustrated graphically for radiation, Casson fluid and time, as well as Magnetohydrodynamics (MHD). It is found that the thermal radiation rises due to an increase in temperature. Besides, the increasing of Casson fluid and MHD parameter has decreasing effect on velocity. Finally, the influence of time will increase the velocity of the fluid.
\end{abstract}

\section{Introduction}

It is well known that non-Newtonian fluids have numerous applications in pharmaceutical, chemical and cosmetic industries such as in the production of gas, paint, oil, juice, syrup, cleanser and several chemicals. In Newtonian fluid, the viscous stresses arising from its flow, at every point are linearly proportional to the local strain rate and their applications are limited, of which many facts are noticed for the fluids in technological and industrial applications such as soap, blood, paints and certain oils are indescribable [1]. Mathematicians, physicists and engineers face a special challenge with the mechanics of non-Newtonian fluids, as the properties of such fluids are unable to describe by Navier-Stokes' equations. Furthermore, there is no equation which exhibits the properties of all the fluids. Several non-Newtonian fluid models have been proposed such as Bingham plastic, power law, Walter-B, viscoplastic, Brinkman type, Oldroyd-B models and Casson fluid due to complex behaviour of fluids [2-8].

\footnotetext{
* Corresponding author.

E-mail address: zulkhibri@ump.edu.my

https://doi.org/10.37934/arfmts.85.1.93100
} 
Casson fluid model was introduced by Casson in 1959 to study the prediction of the flow behaviour of pigment oil suspensions [9]. Khalid et al., [10] considered unsteady boundary layer flow of a Casson fluid past an oscillating vertical plate with constant wall temperature. Nadeem et al., [11] analysed Magnetohydrodynamics (MHD) flow of a Casson fluid over an exponentially shrinking sheet and found that an increase in Casson fluid parameter decreases magnitude of velocity and boundary layer thickness. The study of Newtonian heating effect on unsteady hydromagnetic Casson fluid flow past a flat plate with heat and mass transfer has shown that fluid temperature and velocity decrease with increasing values of Casson parameter while concentration decreases with increasing value of chemical reaction and Schmidt number [12]. Kataria and Patel [13] investigated radiation and chemical reaction effects on MHD Casson fluid flow past an oscillating vertical plate embedded in porous medium. They observed that the fluid is close to the Newtonian fluid for the large value of Casson parameter where the velocity is less than the non-Newtonian fluid. It is seen that the temperature decreases and the velocity increases with increase in thermal radiation and concentration decreases tendency with chemical reaction parameter. Aman et al., [14] examined effect of MHD and porosity on exact solutions and flow of a hybrid Casson nanofluid and figured out that temperature increases with increase of radiation parameter and velocity maximizes with increasing values of Casson parameter and porosity while decreasing with rising values of magnetic parameter.

Mohan et al., [15] presented an unsteady MHD free convection flow of Casson fluid past an exponentially accelerated infinite vertical plate through porous media in the presence of thermal radiation, chemical reaction and heat source or sink. They concluded that the velocity profiles decrease with increasing values of magnetic parameter, Prandtl number, heat source, thermal radiation and Casson parameter. Recently, Deka [16] has conducted a study in presence of thermal radiation through porous medium unsteady MHD Casson fluid past an accelerated vertical plate. It is found that Casson parameter enhances the fluid velocity and skin friction. Furthermore, the surface shear stress increase with the increase in Casson parameter.

Motivated by all the research works that have been done, we consider the effects of radiation and magnetohydrodynamic on unsteady Casson fluid over accelerated plate in this study. Exact solutions are obtained analytically by using Laplace transform. Graphical results are obtained using Mathematica and discussed for various parameters.

\section{Mathematical Formulation and Solution}

In this research, we want to achieve exact solutions for the flow of accelerated Casson fluid with the presence of MHD, radiation and porous medium. We consider unsteady Casson fluid past an accelerated plate situated at the flow being confined to $x>0$, where $x$ is the measure of coordinate in the normal direction to the surface. Initially, for time $t=0$, fluid and plate are both at stationary condition with constant temperature. At $t>0$, the plate is accelerated with velocity $u^{\prime}=A t$. At the same time, the plate temperature $T^{\prime}$ is raised to $T_{w}^{\prime}$, as shown in Figure 1. 


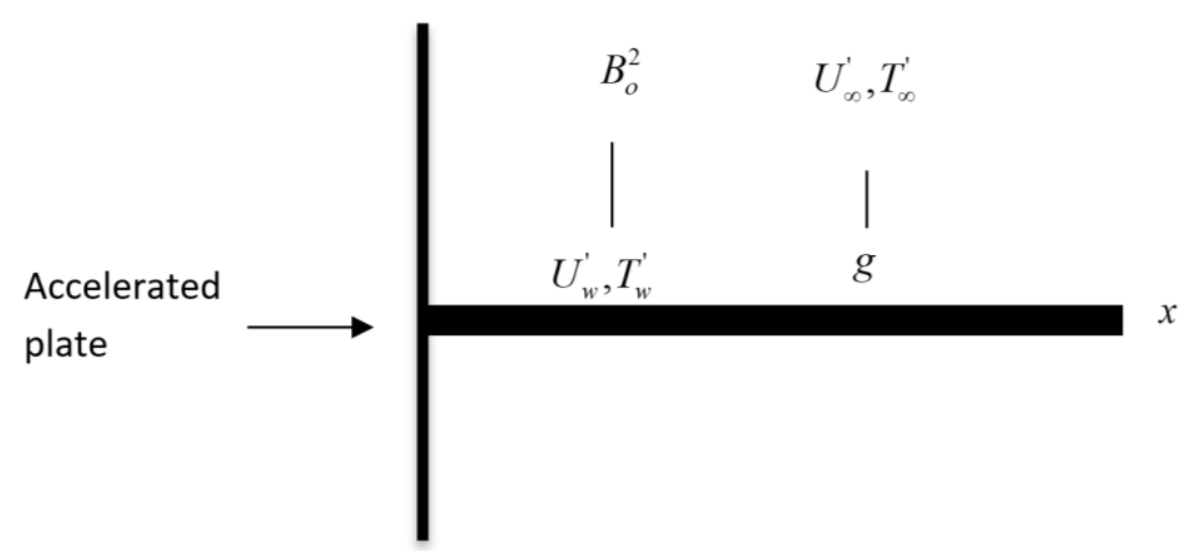

Fig. 1. Geometry configuration and coordinate system

The flow is governed by the following dimensional momentum and energy equations

$\rho \frac{\partial u^{\prime}}{\partial t^{\prime}}=\mu\left(1+\frac{1}{\gamma}\right) \frac{\partial^{2} u^{\prime}}{\partial x^{\prime 2}}+\rho g \beta\left(T^{\prime}-T_{\infty}^{\prime}\right)-\sigma B_{0}^{2} u^{\prime}-\frac{v}{K} u^{\prime}$

$\rho c_{p} \frac{\partial T^{\prime}}{\partial t^{\prime}}=k \frac{\partial^{2} T^{\prime}}{\partial x^{\prime 2}}-\frac{\partial q_{r}^{\prime}}{\partial x^{\prime}}$

Here, $\gamma$ denotes Casson parameter, $u^{\prime}$ represents fluid in the $x$-direction, $t$ refers to time variable, $T^{\prime}$ is temperature of the fluid near the plate, while $T_{\infty}^{\prime}$ represents temperature of the plate, $\rho$ denotes fluid density, $\mu$ is dynamic viscosity, $\beta$ refers to coefficient of the thermal expansion, $B_{\circ}$ is external magnetic field, $v$ represents kinematic viscosity, $k$ denotes thermal conductivity, $K$ refers to porosity, $c_{p}$ is specific heat at constant pressure and $q_{r}$ represents radiative heat flux, along with initial and boundary conditions

$u^{\prime}\left(x^{\prime}, 0\right)=0 ; \quad u^{\prime}\left(0, t^{\prime}\right)=A t ; u^{\prime}\left(\infty, t^{\prime}\right)=0 ;$

$T^{\prime}\left(x^{\prime}, 0\right)=T_{\infty}^{\prime} ; T^{\prime}\left(0, t^{\prime}\right)=T_{w}^{\prime} ; T^{\prime}\left(\infty, t^{\prime}\right)=T_{w}^{\prime}$,

and dimensionless variable

$u=\frac{u^{\prime}}{(v A)^{\frac{1}{3}}} ; t=\frac{t^{\prime} A^{\frac{2}{3}}}{v^{\frac{1}{3}}} ; x=\frac{x^{\prime} A^{\frac{1}{3}}}{v^{\frac{2}{3}}} ; T=\frac{T^{\prime}-T_{\infty}^{\prime}}{T_{w}^{\prime}-T_{\infty}^{\prime}}$.

Rewrite back Eq. (1) and Eq. (2),

$$
\begin{aligned}
& \rho A \frac{\partial u}{\partial t}=\mu\left(1+\frac{1}{\gamma}\right) \frac{A}{v} \frac{\partial^{2} u}{\partial u^{2}}+\rho g \beta\left(T_{W}^{\prime}-T_{\infty}^{\prime}\right)-\sigma B_{\circ}^{2} u(v A)^{\frac{1}{3}}-\frac{v}{k} u(v A)^{\frac{1}{3}}, \\
& \frac{\rho c_{p} v}{k} \frac{\partial T}{\partial t}=\frac{\partial^{2} T}{\partial x^{2}}+\frac{16 \sigma^{*} T_{\infty}^{\prime 3}}{3 k^{*} k} \frac{\partial^{2} T}{\partial x^{2}},
\end{aligned}
$$


where the parameters used in this research are

$G r=\frac{g \beta\left(T_{w}-T_{\infty}\right)}{A} ; N=\frac{16 \sigma^{*} T_{\infty}^{\prime 3}}{3 k k^{*}} ; \operatorname{Pr}=\frac{\mu c_{p}}{k}$.

$G r, N$ and Pr are thermal Grashof number, radiation and Prandtl number. Therefore,

$\frac{\partial u}{\partial t}=\left(1+\frac{1}{\gamma}\right) \frac{\partial^{2} u}{\partial x^{2}}+G r T-\sigma B_{\circ}^{2} \frac{u v^{\frac{1}{3}}}{\rho A^{\frac{2}{3}}}-\frac{v}{k} \frac{u v^{\frac{1}{3}}}{\rho A^{\frac{2}{3}}}$,

$\frac{\partial T}{\partial t}=\frac{(1+N)}{\operatorname{Pr}} \frac{\partial^{2} \bar{T}}{\partial x^{2}}$

By using Laplace transform for Eq. (8) and Eq. (9),

$$
\begin{aligned}
& \left(1+\frac{1}{\gamma}\right) \frac{d^{2} \bar{u}}{d x^{2}}-\left(s+\frac{\sigma B_{\circ}^{2} v^{\frac{1}{3}}}{\rho A^{\frac{2}{3}}}+\frac{v}{k} \frac{v^{\frac{1}{3}}}{\rho A^{\frac{2}{3}}}\right) \bar{u}=-G r \bar{T}, \\
& \frac{d^{2} T}{d x^{2}}-a s \bar{T}=0, \quad a=\frac{\operatorname{Pr}}{1+N} .
\end{aligned}
$$

Applying the inverse Laplace transform emits

$$
\begin{aligned}
& u(x, t)= \frac{1}{2} e^{x \sqrt{z L}} \operatorname{erfc}\left(\frac{x}{2} \sqrt{\frac{z}{t}}+\sqrt{L t}\right)+e^{-x \sqrt{L z}} \operatorname{erfc}\left(\frac{x}{2} \sqrt{\frac{z}{t}}-\sqrt{L t}\right)+ \\
& {\left[-\frac{1}{2 j} e^{x \sqrt{z L}} \operatorname{erfc}\left(\frac{x}{2} \sqrt{\frac{z}{t}}-\sqrt{L t}\right)+e^{-x \sqrt{L z}} \operatorname{erfc}\left(\frac{x}{2} \sqrt{\frac{z}{t}}-\sqrt{L t}\right)\right]+} \\
& \frac{1}{j} \operatorname{erfc}\left(\frac{x}{2} \sqrt{\frac{9}{t}}\right)+\left[\frac{e^{j t}}{2 j} e^{x \sqrt{z(L+j)}} \operatorname{erfc}\left(\frac{x}{2} \sqrt{\frac{z}{t}}+\sqrt{t(L+j)}\right)+\right. \\
& e^{-x \sqrt{z(L+j)}} \operatorname{erfc}\left(\frac{x}{2} \sqrt{\frac{z}{t}}-\sqrt{t(L+j)}\right)+\left[-\frac{1}{j} \frac{e^{j t}}{2} e^{x \sqrt{a j}} \operatorname{erfc}\left(\frac{x}{2} \sqrt{\frac{a}{t}}+\sqrt{j t}\right)+\right. \\
&\left.e^{-x \sqrt{a j}} \operatorname{erfc}\left(\frac{x}{2} \sqrt{\frac{a}{t}}-\sqrt{j t}\right)\right] \\
& T(x, t)=\operatorname{erfc}\left(\frac{x \sqrt{a}}{2 \sqrt{t}}\right)
\end{aligned}
$$

\section{Numerical Results and Discussions}

In this section, we discuss the different physical parameters such as thermal radiation, Casson parameter, time and magnetic parameter. Figure 2 shows the temperature profiles of thermal radiation for the fixed values of $\operatorname{Pr}=20$ and $t=0.5$ with different values of $N=3,5,7$ and 9 . It is seen 
that the thermal radiation rises due to an increase in temperature. The increasing radiation parameter increases heat absorption and results in rise in the fluid temperature.

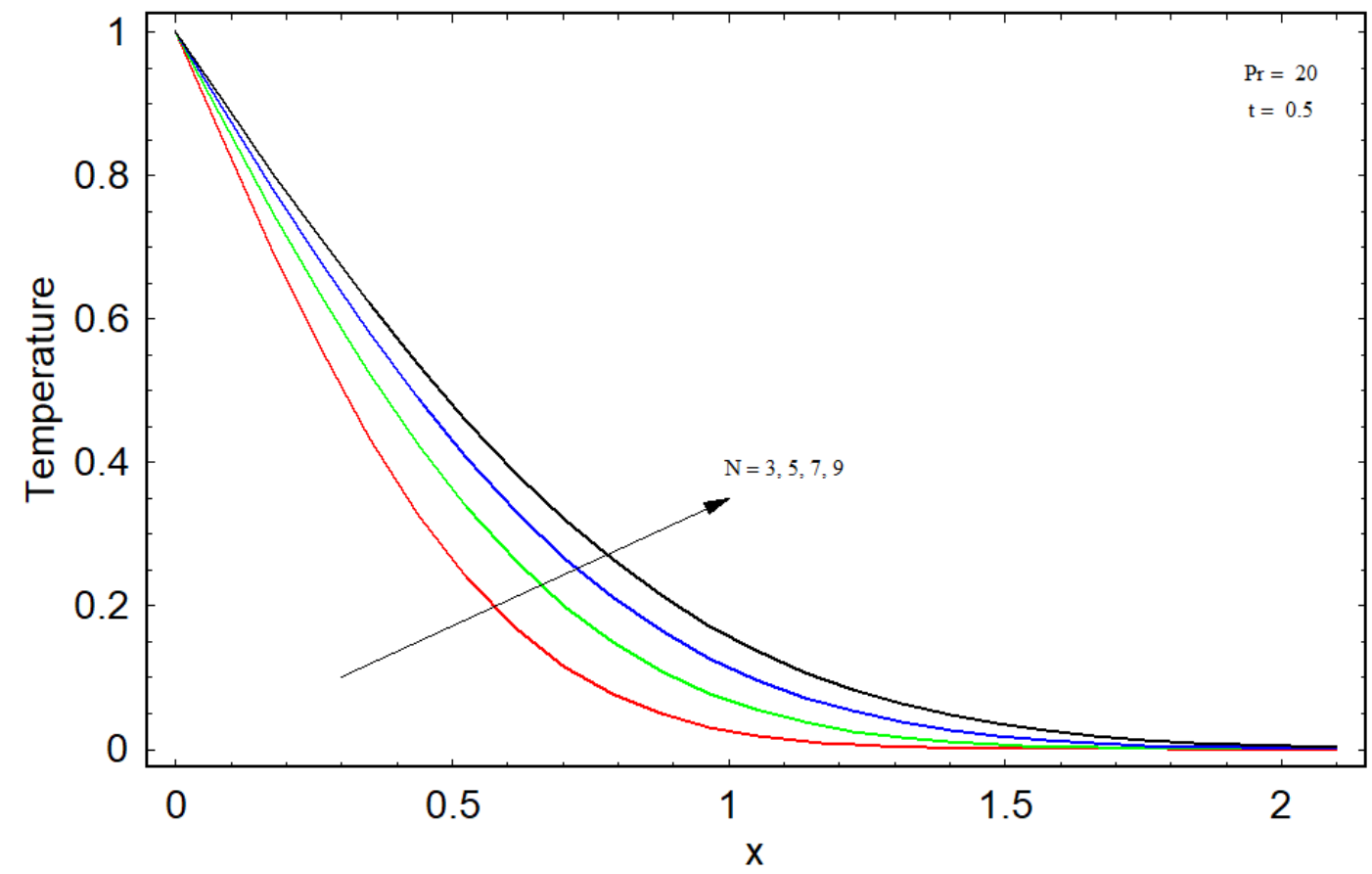

Fig. 2. Temperature profiles for different values of $\mathrm{N}$

Figure 3 illustrates the velocity profiles for the fixed values of $\operatorname{Pr}=20, t=0.5, G r=1, N=3, M=5$ and $K=0.3$ with different values of $\gamma=0.2,0.3,0.4$ and 0.5 . It is observed that decreasing fluid velocity is due to increasing in Casson parameter. This behaviour indicates that higher values of Casson parameter increase the plastic dynamic viscosity of the fluid. This phenomenon creates resistance in the flow of fluid and thus, a decrease in fluid velocity is observed.

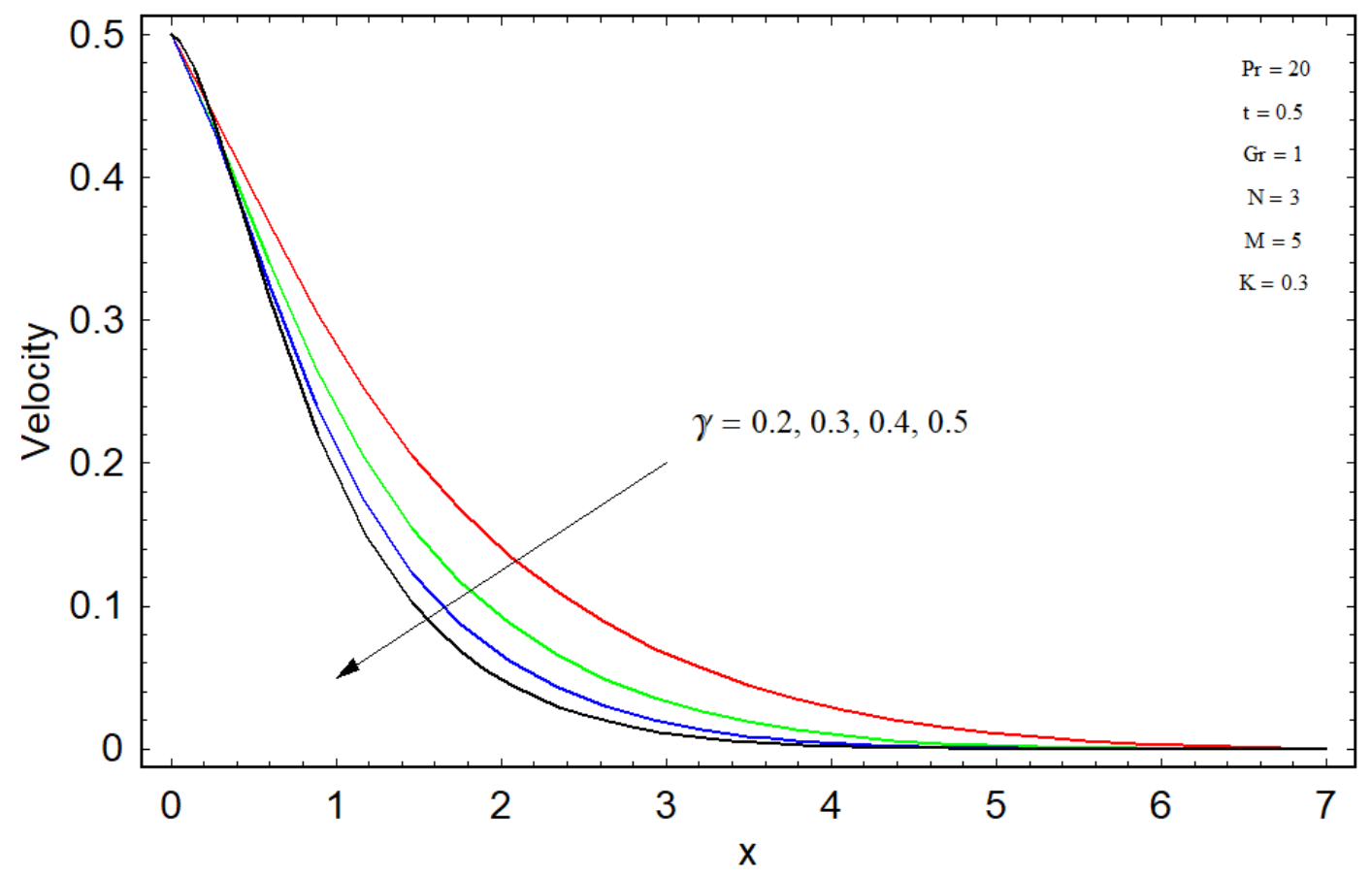

Fig. 3. Velocity profile for different values of $\gamma$ 
Figure 4 depicts the velocity profile for time parameter with fixed values of $\operatorname{Pr}=20, \gamma=0.2, G r=1, N=3, M=5$ and $K=0.3$ with different values of $t=0.3,0.5,0.7$ and 1 . Time will increase the velocity of the fluid as displayed in Figure 4. Figure 5 is plotted for velocity profile of magnetic parameter with fixed values of $\operatorname{Pr}=20, t=0.5, \gamma=0.2, G r=1, N=3$, and $K=0.3$ with different values of $M=5,7,9$ and 11 . It is found that magnetic parameter will decrease the velocity of the fluid. An increase in the values of magnetic parameter will reduce velocity boundary layer. This occurs due to an increase in magnetic field that develops the opposite force to the flow direction. It is called Lorentz force and it has the tendency to reduce the boundary layer thickness.

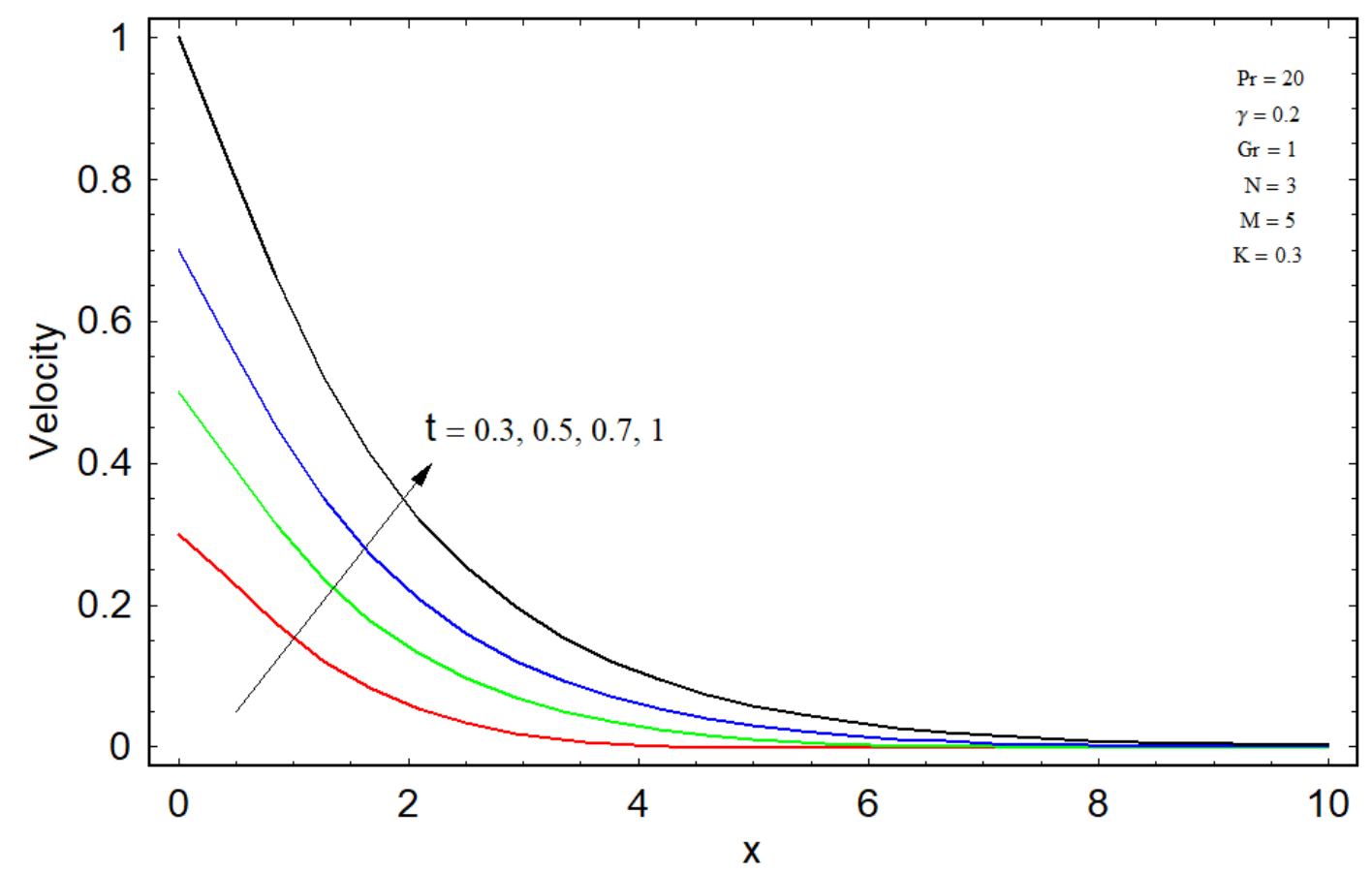

Fig. 4. Velocity profile for different values of $t$

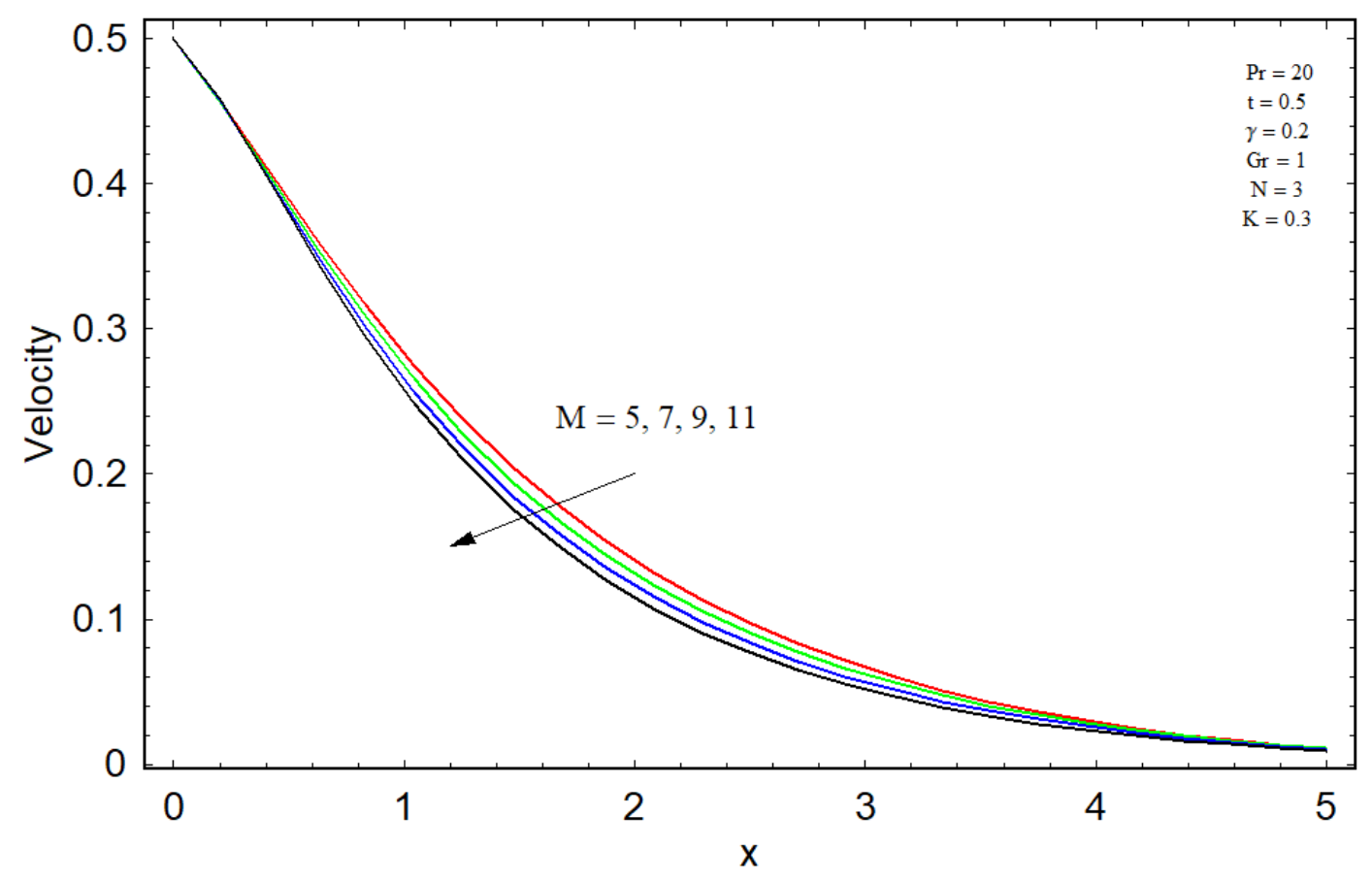

Fig. 5. Velocity profile for different values of $M$ 


\section{Conclusions}

The effects of porosity and radiation on unsteady Casson fluid over accelerated plate are obtained. The solution is derived by using Laplace transform technique. Graphical results for temperature and velocity are discussed in detail. It is found that an increase in thermal radiation leads to an increase in temperature. Besides, the increasing of Casson fluid and MHD has decreasing effect on velocity. Finally, the increasing velocity of the fluid is due to an increase in time.

\section{Acknowledgement}

The authors would like to acknowledge Universiti Malaysia Pahang (Pusat Bendalir UMP) for the financial support through vote numbers RDU190376 for this research.

\section{References}

[1] Chaoyang, Wang, and Tu Chuanjing. "Boundary-layer flow and heat transfer of non-Newtonian fluids in porous media." International Journal of Heat and Fluid Flow 10, no. 2 (1989): 160-165. https://doi.org/10.1016/0142727X(89)90011-8

[2] Kleppe, J., and W. J. Marner. "Transient free convection in a Bingham plastic on a vertical flat plate." Journal of Heat Transfer 94, no. 4 (1972): 371-376. https://doi.org/10.1115/1.3449954

[3] Olajuwon, B. I. "Flow and natural convection heat transfer in a power law fluid past a vertical plate with heat generation." International Journal of Nonlinear Science 7, no. 1 (2009): 50-56.

[4] Khan, Ilyas, Farhad Ali, Sharidan Shafie, and Muhammad Qasim. "Unsteady free convection flow in a Walters-B fluid and heat transfer analysis." Bulletin of the Malaysian Mathematical Sciences Society 37, no. 2 (2014): 437-448.

[5] Hassan, M. A., Manabendra Pathak, and Mohd Khan. "Natural convection of viscoplastic fluids in a square enclosure." Journal of Heat Transfer 135, no. 12 (2013): 122501. https://doi.org/10.1115/1.4024896

[6] Khan, Ilyas, Kamran Fakhar, and Muhammad Imran Anwar. "Hydromagnetic rotating flows of an Oldroyd-B fluid in a porous medium." Special Topics \& Reviews in Porous Media: An International Journal 3, no. 1 (2012): 89-95. https://doi.org/10.1615/SpecialTopicsRevPorousMedia.v3.i1.80

[7] Alkasasbeh, Hamzeh Taha. "Numerical solution of micropolar Casson fluid behaviour on steady MHD natural convective flow about a solid sphere." Journal of Advanced Research in Fluid Mechanics and Thermal Sciences 50, no. 1 (2018): 55-66.

[8] Jamil, Dzuliana Fatin, Uddin, Salah and Roslan, Rozaini. "The Effects of Magnetic Casson Blood Flow in an Inclined Multi-stenosed Artery by using Caputo-Fabrizio Fractional Derivatives." Journal of Advanced Research in Materials Science 72, no. 1 (2020): 15-30.

[9] Casson, N. "A Flow Equation for Pigment-Oil Suspensions of the Printing Ink Type." Rheology of Disperse Systems (1959).

[10] Khalid, Asma, llyas Khan, and Sharidan Shafie. "Unsteady boundary layer flow of a Casson fluid past an oscillating vertical plate with constant wall temperature." Malaysian Journal of Fundamental and Applied Sciences 11, no. 1 (2015): 28-32. https://doi.org/10.11113/mjfas.v11n1.360

[11] Nadeem, Sohail, Rizwan UI Haq, and C. Lee. "MHD flow of a Casson fluid over an exponentially shrinking sheet." Scientia Iranica 19, no. 6 (2012): 1550-1553. https://doi.org/10.1016/i.scient.2012.10.021

[12] Das, M., R. Mahato, and R. Nandkeolyar. "Newtonian heating effect on unsteady hydromagnetic Casson fluid flow past a flat plate with heat and mass transfer." Alexandria Engineering Journal 54, no. 4 (2015): 871-879. https://doi.org/10.1016/j.aej.2015.07.007

[13] Kataria, Hari R., and Harshad R. Patel. "Radiation and chemical reaction effects on MHD Casson fluid flow past an oscillating vertical plate embedded in porous medium." Alexandria Engineering Journal 55, no. 1 (2016): 583-595. https://doi.org/10.1016/i.aej.2016.01.019

[14] Aman, Sidra, Syazwani Mohd Zokri, Zulkhibri Ismail, Mohd Zuki Salleh, and Ilyas Khan. "Effect of MHD and porosity on exact solutions and flow of a hybrid Casson-nanofluid." Journal of Advanced Research in Fluid Mechanics and Thermal Sciences 44, no. 1 (2018): 131-139.

[15] Mohan, S. Rama, G. Viswanatha Reddy, and S. Balakrishna. "An unsteady MHD free convection flow of casson fluid past an exponentially accelerated infinite vertical plate through porous media in the presence of thermal radiation, chemical reaction and heat source or sink." International Journal of Engineering and Techniques 4, no. 4 (2018): 1627. 
[16] Deka, Anjan Kumar. "In presence of thermal radiation through porous medium unsteady MHD Casson fluid flow past an accelerated vertical plate." International Journal of Statistics and Applied Mathematics 5, no. 4 (2020): 213228. 